Virginia Commonwealth University VCU Scholars Compass

2010

\title{
Probing the existence of energetically degenerate cluster isomers by chemical tagging
}

Qian Wang

Virginia Commonwealth University, qwang@vcu.edu

Qiang Sun

Virginia Commonwealth University, Peking University

Puru Jena

Virginia Commonwealth University, pjena@vcu.edu

Follow this and additional works at: http://scholarscompass.vcu.edu/phys_pubs

Part of the Physics Commons

Wang, Q. Sun, Q. Jena, P. Probing the existence of energetically degenerate cluster isomers by chemical tagging. Applied Physics Letters, 97, 223104 (2010). Copyright (C) 2010 AIP Publishing LLC.

\section{Downloaded from}

http://scholarscompass.vcu.edu/phys_pubs/31

This Article is brought to you for free and open access by the Dept. of Physics at VCU Scholars Compass. It has been accepted for inclusion in Physics Publications by an authorized administrator of VCU Scholars Compass. For more information, please contact libcompass@vcu.edu. 


\title{
Probing the existence of energetically degenerate cluster isomers by chemical tagging
}

\author{
Qian Wang, ${ }^{1, a)}$ Qiang Sun, ${ }^{1,2}$ and Puru Jena ${ }^{1}$ \\ ${ }^{1}$ Department of Physics, Virginia Commonwealth University, Richmond, Virginia 23284, USA \\ ${ }^{2}$ Department of Advanced Materials and Nanotechnology and Center for Applied Physics and Technology, \\ Peking University, Beijing 1000871, People's Republic of China
}

(Received 25 August 2010; accepted 5 November 2010; published online 30 November 2010)

\begin{abstract}
Current methods for identifying the ground state geometry of a cluster require synergy between theory and experiment. However, this becomes a difficult problem when the accuracy of the theoretical methods is not sufficient to distinguish between nearly degenerate isomers. Using density functional theory based calculations, we show that the near degeneracy between the planar and the cage structures can be lifted by tagging these with halogens and superhalogens moieties such as $\mathrm{Cl}$ and $\mathrm{BO}_{2}$. The energy of the planar $\mathrm{Au}_{16}{ }^{-}$isomer is lowered from $0.15 \mathrm{eV}$ before tagging to 0.51-0.55 eV after tagging, thus providing a way to probe its coexistence. (C) 2010 American Institute of Physics. [doi:10.1063/1.3521282]
\end{abstract}

Current methods for obtaining cluster geometries, rely on a synergy between theory and experiment where structures determined from first-principles calculations are validated by comparing computed properties with experiments such as photoelectron spectroscopy (PES), ${ }^{1-3}$ trapped ion electron diffraction, ${ }^{4}$ ion mobility, ${ }^{5,6}$ and infrared absorption spectroscopy. ${ }^{7}$ While this scheme has been useful in elucidating the structures of numerous clusters, serious limitations arise when the lowest energy structures have nearly degenerate isomers and limited accuracy of the state-of-the-art theoretical techniques does not permit a way to distinguish between them. A case in point is the $\mathrm{Au}_{16}{ }^{-}$cluster. In combination with theory, several experiments have recently identified its geometry to be a hollow cage with $\mathrm{T}_{d}$ symmetry. This has attracted considerable attention not only because it is the metallic cluster behaving like a cousin of the $\mathrm{C}_{60}$ fullerene but also because the space inside the hollow cage is large enough to accommodate guest atoms and hence can have technological applications. A recent theoretical calculation by Xing et al. ${ }^{4}$ as well that performed in our group ${ }^{8}$ have identified a planar $\mathrm{Au}_{16}{ }^{-}$that lies lower in energy than the hollow cage structure by $0.08-0.22 \mathrm{eV}$, respectively, depending on the calculation method. Second, the PES data ${ }^{1,3}$ show that the vertical and adiabatic detachment energies of $\mathrm{Au}_{16}{ }^{-}$are nearly identical. This implies that the geometries of anionic and neutral $\mathrm{Au}_{16}$ cluster are the same. However, theoretical calculations done in our group ${ }^{8}$ as well those published earlier ${ }^{9,10}$ show that the two geometries are rather different and the neutral $\mathrm{Au}_{16}$ has a compact structure. In addition, the planar geometry of neutral $\mathrm{Au}_{16}$ cluster is nearly degenerate with compact three dimensional geometry. Yet, no experiments have identified this planar structure. The question then remains: is there a way to lift the degeneracy between the planar and the cage structures and thus make it easier to observe the planar isomer? In this paper, we show that this indeed can be achieved by suitable tagging. We show that by reacting $\mathrm{Au}_{16}{ }^{-}$with either $\mathrm{Cl}$ or superhalogen $\mathrm{BO}_{2}$, the energy of the planar structure can be lowered by as much as $0.50 \mathrm{eV}$. Equally important, the geometry of the

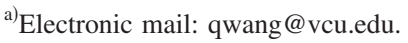

$\mathrm{Au}_{16}{ }^{-}$cluster remains essentially the same after tagging. Thus, chemical tagging can be used to distinguish between energetically degenerate isomers just as tagging with rare gas atoms has recently been used ${ }^{11}$ to study two dimensional (2D) to three dimensional (3D) transition in the Au clusters.

In choosing an appropriate tagging agent, we recall a recent experiment involving the interaction of the Au clusters with $\mathrm{BO}_{2}$ moieties in a pulsed arc cluster ion source. ${ }^{12}$ Here, it was found that the electronic properties of bare Au clusters, namely, the odd-even alternation in the electron affinity, remained unchanged after reaction. Accompanying theoretical calculations ${ }^{13}$ showed that the geometries of the Au clusters remained mostly unaltered. This was shown to be a consequence of the superhalogen property of $\mathrm{BO}_{2}$. For example, the electron affinity of $\mathrm{BO}_{2}$, namely, $4.46 \mathrm{eV}^{13}$ is much larger than that of $\mathrm{Cl}$, which is $3.6 \mathrm{eV}$. Since the electron affinity of $\mathrm{BO}_{2}$ is larger than that of the $\mathrm{Au}_{n}$ clusters, an electron from $\mathrm{Au}_{n}$ is transferred to the $\mathrm{BO}_{2}$ moiety, thus leaving these clusters positively charged. As an extra electron is attached to the $\mathrm{Au}_{n} \mathrm{BO}_{2}$ clusters, it neutralizes the positive charge on the $\mathrm{Au}_{n}$ portion of the $\mathrm{Au}_{n} \mathrm{BO}_{2}$ cluster, and hence, the $\mathrm{Au}_{n}$ cluster retains its initial structure. We, therefore, tagged both neutral and anionic $\mathrm{Au}_{16}$ clusters with $\mathrm{BO}_{2}$ moiety and optimized the resulting geometries, total energies, and spectroscopic properties. We concentrated on two main isomers of $\mathrm{Au}_{16}$, namely, the planar and hollow cage structures. The structures of the $\mathrm{Au}_{16}{ }^{-}$isomers were found to be unchanged after tagging even if the $\mathrm{BO}_{2}$ moiety is bound to the planar and cage isomers with binding energies of 3.54 and $3.14 \mathrm{eV}$, respectively. The calculations were repeated by tagging the $\mathrm{Au}_{16}$ isomers with $\mathrm{Cl}$ atom since the electron affinity of $\mathrm{Cl}$ is also higher than those in the $\mathrm{Au}_{n}$ clusters. The energy of the anion planar isomer is lowered from $0.15 \mathrm{eV}$ before tagging to $051-0.55 \mathrm{eV}$ after tagging, depending on whether $\mathrm{Cl}$ or $\mathrm{BO}_{2}$ moiety is used. In the following, we present the details of our calculations and the results.

The calculations were carried out using density functional theory and generalized gradient approximation for exchange and correlation with PW91 functional. ${ }^{14}$ We used plane-wave basis set with the projector augmented wave 


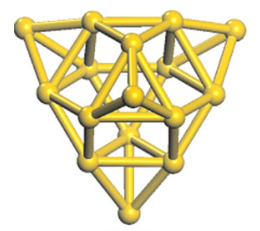

$\left(N_{1}\right)$

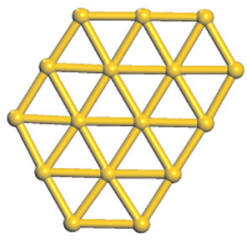

$\left(N_{2}\right)$

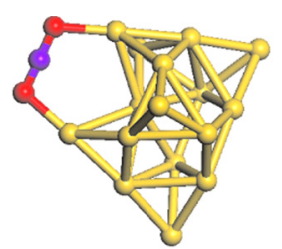

$\left(N_{1 \mathrm{~B}}\right)$

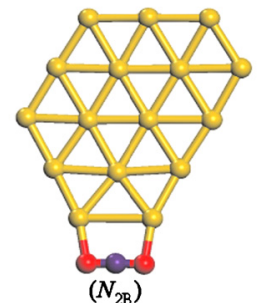

$\left(N_{2 \mathrm{~B}}\right)$

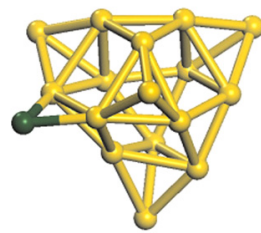

$\left(N_{1 \mathrm{C}}\right)$

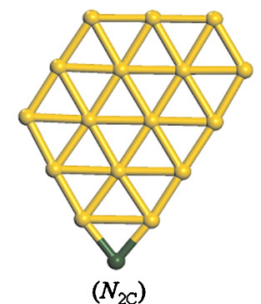

$\left(N_{2 C}\right)$
FIG. 1. (Color online) $\left(N_{1}\right)$ and $\left(N_{2}\right)$ are two of the lowest lying isomers of the neutral $\mathrm{Au}_{16}$ cluster. $\left(N_{1 B}\right)$ and $\left(N_{2 B}\right)$ represent the corresponding lowest energy configurations after $\mathrm{BO}_{2}$ is attached. $\left(N_{1 C}\right)$ and $\left(N_{2 C}\right)$ are the corresponding lowest energy isomers after $\mathrm{Cl}$ is attached.

method $^{15}$ as implemented in the Vienna ab initio simulation package (VASP). ${ }^{16}$ We used the supercell approach where clusters were placed at the center of the $24 \times 24 \times 24 \AA^{3}$ cubic cell. The geometries were optimized without any symmetry constraint and high precision calculations with a cutoff energy of $400 \mathrm{eV}$ for the plane-wave basis were performed. Due to the large supercell, the Brillouin zone integration was performed only at the $\Gamma$ point. In all calculations, selfconsistency was achieved with a tolerance in the total energy of at least $0.2 \mathrm{meV}$. Hellman-Feynman force components on each ion in the supercells were converged to $2 \mathrm{meV} / \AA$.

In Fig. 1, we present the geometries of neutral $\mathrm{Au}_{16}$ cluster. The compact isomer with $T_{\mathrm{d}}$ symmetry, labeled $N_{1}$, is $0.14 \mathrm{eV}$ lower in energy than the planar isomer, labeled $N_{2}$. Note that Chen et al. ${ }^{8}$ found the 3D compact structure to be $0.12 \mathrm{eV}$ lower in energy than the $2 \mathrm{D}$ planar one. Within the accuracy of DFT calculations, these two isomers can be regarded as energetically nearly degenerate. To compute the geometries of the $\mathrm{Au}_{16}$ cluster after $\mathrm{BO}_{2}$ or $\mathrm{Cl}$ is attached, we carried out extensive geometry optimization by considering all the possible binding configurations. To facilitate the choice of initial configurations, we calculated the charge on each of the $\mathrm{Au}$ atoms in the $\mathrm{Au}_{16}$ cluster using Bader charge analysis ${ }^{17,18}$ and attached $\mathrm{BO}_{2}$ or $\mathrm{Cl}$, starting with the atoms carrying more positive charge. We found that the corner edge $\mathrm{Au}$ atoms are more reactive than others and preferably bind to $\mathrm{BO}_{2}$ and $\mathrm{Cl}$. The final geometries given in Fig. 1 are labeled $N_{1 B}, N_{1 C}, N_{2 B}$, and $N_{2 C}$, respectively, and $B$ and $C$ represent $\mathrm{Au}_{16} \mathrm{BO}_{2}$ and $\mathrm{Au}_{16} \mathrm{Cl}$ clusters. The binding energies of neutral $\mathrm{Au}_{16}$ in the 3D compact structure with $\mathrm{BO}_{2}$ and $\mathrm{Cl}\left[\left(N_{1 B}\right)\right.$ and $\left(N_{1 C}\right)$ in Fig. 1] were calculated to be 2.87 and $2.58 \mathrm{eV}$, respectively, while those for the planar structures $\left[\left(N_{2 B}\right)\right.$ and $\left(N_{2 C}\right)$ in Fig. 1] are 3.03 and $3.02 \mathrm{eV}$. The $\mathrm{Au}-\mathrm{O}$ bonding distances between the $\mathrm{Au}_{16}$ cluster and the $\mathrm{BO}_{2}$ molecule in the $3 \mathrm{D}$ and $2 \mathrm{D}$ structures are 2.19 and 2.13 $\AA$, respectively. Tagging slightly elongates the bond length of the $\mathrm{BO}_{2}$ molecule where the $\mathrm{B}-\mathrm{O}$ bond length increases to 1.28 and $1.29 \AA$ for the $3 \mathrm{D}$ and $2 \mathrm{D}$ structures, respectively, from $1.26 \AA$ in its free state. For $\mathrm{Cl}$ tagging, the $\mathrm{Au}-\mathrm{Cl}$ bonding distances in the $3 \mathrm{D}\left(N_{1 C}\right)$ and $2 \mathrm{D}\left(N_{2 C}\right)$ isomers are 2.45 and $2.43 \AA$, respectively. We note that the bonding distance in the 3D structure for both $\mathrm{BO}_{2}$ and $\mathrm{Cl}$ tagging is larger than that in the $2 \mathrm{D}$ structure. Consequently the bind-
TABLE I. The calculated relative energies $\Delta E$ (in $\mathrm{eV}$ ) of isomers $\left(N_{1}\right.$ and $N_{2}$ ) of the $\mathrm{Au}_{16}$ cluster tagging with $\mathrm{BO}_{2}$ and $\mathrm{Cl}$. The energies are measured with respect to the lowest energy structure for which $\Delta E$ is marked as 0.00 eV. Also given are the HOMO-LUMO gaps $\left(\Delta_{\text {gap }}\right.$ in $\left.\mathrm{eV}\right)$, and the first VIPs (in $\mathrm{eV}$ ) for the compact cage and planar isomers of the neutral $\mathrm{Au}_{16} \mathrm{BO}_{2}$ and $\mathrm{Au}_{16} \mathrm{Cl}$ clusters (see Fig. 1).

\begin{tabular}{lccc}
\hline \hline \multicolumn{1}{c}{ Isomers } & $\Delta E$ & $\Delta_{\text {gap }}$ & VIP \\
\hline$N_{1 B}$ & 0.00 & 0.12 & 5.84 \\
$N_{2 B}$ & 0.16 & 0.11 & 6.25 \\
$N_{1 C}$ & 0.44 & 0.12 & 5.71 \\
$\mathrm{~N}_{2 C}$ & 0.00 & 0.11 & 6.10 \\
\hline \hline
\end{tabular}

ing between the attached molecules and the neutral $\mathrm{Au}_{16}$ in the 3D structures is weaker than that in the planar structures. The calculated relative binding energies are given in Table I. The following observations can be made from Fig. 1 and Table I: (1) geometries of the $\mathrm{Au}_{16}$ isomers remain relatively undisturbed after tagging, although change in the structure due to $\mathrm{BO}_{2}$ attachment is more that than when $\mathrm{Cl}$ is attached. (2) While isomer $\mathrm{N}_{1 B}$ in Fig. 1 is lower in energy than isomer $\mathrm{N}_{2 B}$ by only $0.16 \mathrm{eV}$, the energy ordering of $N_{1 C}$ and $N_{2 C}$ is reversed; here, the 2D structure becomes $0.44 \mathrm{eV}$ lower in energy than the 3D one. The energy degeneracy between the isomers in the planar structure is lifted in three dimensions. (3) Among all the four geometries, although 2D isomers have almost the same highest occupied molecular orbital (HOMO) and lowest occupied molecular orbital (LUMO) gap (about $0.1 \mathrm{eV}$ ) as the 3D isomers, the first vertical ionization potentials (VIPs) of the 2D isomers are about $0.40 \mathrm{eV}$ higher than those of the 3D isomers.

The results for the $\mathrm{Au}_{16}$ anionic cluster are given in Fig. 2 and Table II. The planar $\mathrm{Au}_{16}$ isomer (labeled $A_{1}$ ) is found to be $0.15 \mathrm{eV}$ lower in energy than the hollow cage structure $\left(A_{2}\right)$. Note that Xing et al. ${ }^{4}$ and Chen et $_{\text {al. }}{ }^{8}$ found the planar structures to be 0.08 and $0.22 \mathrm{eV}$ lower in energy than the hollow cage structure, respectively. We optimized the geometries of the $\mathrm{Au}_{16} \mathrm{BO}_{2}$ and $\mathrm{Au}_{16} \mathrm{Cl}$ anions again by considering all the possible binding sites. We found that for the $2 \mathrm{D}$ isomer $\left(A_{1}\right)$, the corner edge sites are much more reactive than other sites. The resulting geometries of $\mathrm{Au}_{16} \mathrm{BO}_{2}^{-}$and $\mathrm{Au}_{16} \mathrm{Cl}^{-}$anions, labeled $A_{1 B}$ and $A_{1 C}$, are shown in Fig. 2. For the cage isomer $\left(A_{2}\right)$ of $\mathrm{Au}_{16}{ }^{-}$, the four triangle faces are found to be more reactive than other sites. The lowest energy configurations of $\mathrm{Au}_{16} \mathrm{BO}_{2}^{-}$and $\mathrm{Au}_{16} \mathrm{Cl}^{-}$starting from the

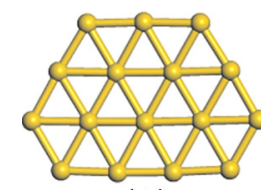

$\left(A_{1}\right)$

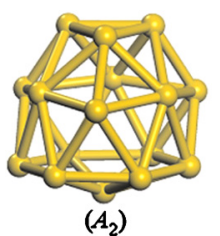

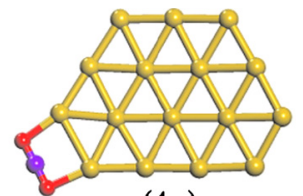

$\left(A_{1 \mathrm{~B}}\right)$

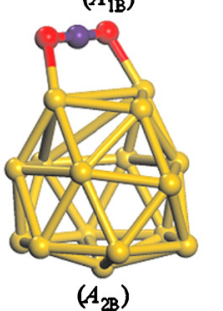

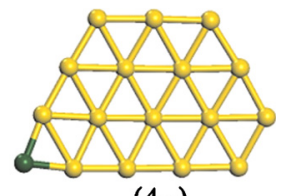

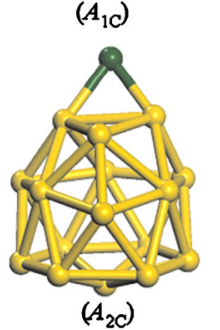

FIG. 2. (Color online) $\left(A_{1}\right)$ and $\left(A_{2}\right)$ are two of the lowest lying isomers of the anionic $\mathrm{Au}_{16}$ cluster. $\left(A_{1 B}\right)$ and $\left(A_{2 B}\right)$ represent the corresponding lowest energy configurations after $\mathrm{BO}_{2}$ is attached. $\left(A_{1 C}\right)$ and $\left(A_{2 C}\right)$ are the corresponding lowest energy isomers after $\mathrm{Cl}$ is attached. 
TABLE II. The calculated relative energies $\Delta E$ (in $\mathrm{eV}$ ) of isomers $\left(A_{1}\right.$ and $A_{2}$ ) of the $\mathrm{Au}_{16}$ anion cluster tagging with $\mathrm{BO}_{2}$ and $\mathrm{Cl}$. The energies are measured with respect to the lowest energy structure for which $\Delta E$ is marked as $0.00 \mathrm{eV}$. Also given are the HOMO-LUMO gaps $\left(\Delta_{\text {gap }}\right.$ in $\left.\mathrm{eV}\right)$, and the first VDEs (in eV) for the hollow cage and planar isomers of the anionic $\mathrm{Au}_{16} \mathrm{BO}_{2}^{-}$and $\mathrm{Au}_{16} \mathrm{Cl}^{-}$clusters (see Fig. 2).

\begin{tabular}{lccc}
\hline \hline Isomers & $\Delta E$ & $\Delta_{\text {gap }}$ & VDE \\
\hline$A_{1 \mathrm{~B}}$ & 0.00 & 0.78 & 4.92 \\
$A_{2 \mathrm{~B}}$ & 0.55 & 0.84 & 4.91 \\
$A_{1 \mathrm{C}}$ & 0.00 & 0.75 & 4.79 \\
$A_{2 \mathrm{C}}$ & 0.51 & 0.77 & 4.76 \\
\hline \hline
\end{tabular}

cage structure of $A_{2}$ are also shown in Fig. 2. These are labeled $A_{2 B}$ and $A_{2 C}$, respectively. Our observations can be summarized as follows: (1) degeneracy in energy between the planar and the cage isomers of the $\mathrm{Au}_{16}{ }^{-}$cluster is lifted. For the bare $\mathrm{Au}_{16}$ anion, the energy difference between the planar and the hollow cage is only $0.15 \mathrm{eV}$, which increases to 0.55 and $0.51 \mathrm{eV}$ after attaching $\mathrm{BO}_{2}$ and $\mathrm{Cl}$, respectively. (2) The reaction with $\mathrm{BO}_{2}$ and $\mathrm{Cl}$ has little effect on the HOMO-LUMO gaps, which are 0.78 and $0.84 \mathrm{eV}$ for the 2D $\left(A_{1 B}\right)$ and $3 \mathrm{D}\left(A_{2 B}\right) \mathrm{Au}_{16} \mathrm{BO}_{2}{ }^{-}$isomers, and 0.75 and $0.77 \mathrm{eV}$ for the 2D $\left(A_{1 C}\right)$ and 3D $\left(A_{2 C}\right) \mathrm{Au}_{16} \mathrm{Cl}^{-}$isomers. (3) The first vertical detachment energies (VDEs) for the 2D and the hollow cage $\mathrm{Au}_{16} \mathrm{BO}_{2}^{-}$isomers are 4.92 and $4.90 \mathrm{eV}$, respectively, while these are 4.79 and $4.76 \mathrm{eV}$ for the $2 \mathrm{D}$ and the hollow cage $\mathrm{Au}_{16} \mathrm{Cl}^{-}$isomers. While these energy differences between $2 \mathrm{D}$ and hollow cage isomers may be difficult to distinguish from photoelectron spectra, totally different shapes of 2D and hollow cage isomers should enable ion mobility experiment to detect the planar isomer tagged with $\mathrm{BO}_{2}$ or $\mathrm{Cl}$ as they are thermodynamically more stable. (4) Compared to the neutral clusters, the geometry distortions of anion are much less when $\mathrm{BO}_{2}$ and $\mathrm{Cl}$ are introduced. The $\mathrm{B}-\mathrm{O}$ bond lengths in both the planar and cage isomers of anionic $\mathrm{Au}_{16} \mathrm{BO}_{2}^{-}$are nearly identical at $1.28 \AA$. The $\mathrm{Au}-\mathrm{O}$ bonding distance, however, is slightly different: $2.22 \AA$ in the planar structure and $2.26 \AA$ in the cage structure. Similarly, the $\mathrm{Au}-\mathrm{Cl}$ bonding distance of $2.37 \AA$ in the $2 \mathrm{D}$ isomer is shorter compared to $2.60 \AA$ in the $3 \mathrm{D}$ structure. (5) The binding energies of anionic $\mathrm{Au}_{16}{ }^{-}$planar structure with $\mathrm{BO}_{2}$ and $\mathrm{Cl}$ are 3.54 and $3.57 \mathrm{eV}$, respectively, while those for the hollow cage structure $\mathrm{Au}_{16} \mathrm{BO}_{2}{ }^{-}$and $\mathrm{Au}_{16} \mathrm{Cl}^{-}$are 3.14 and $3.22 \mathrm{eV}$, respectively.

The changes in the electronic structure of the $\mathrm{Au}_{16}$ clusters after being tagged by $\mathrm{BO}_{2}$ and $\mathrm{Cl}$ can also be seen by examining their respective frontier HOMO and LUMO orbitals. These are plotted in Fig. 3. Note that the $5 d$ orbital characteristics of $\mathrm{Au}$ are enhanced after tagging, and the orbital distributions become more homogeneous. We conclude from the above results that tagging with $\mathrm{BO}_{2}$ and $\mathrm{Cl}$ not only lifts the energy degeneracy between the planar and hollow cage isomers for the anion but also enhances the energetic stability of the planar isomer.

In summary, we have shown that by chemical tagging, it is possible to distinguish between nearly degenerate isomers of their host cluster as one may be able to thermodynamically stabilize one isomer against another. Even if the binding energies of $\mathrm{BO}_{2}$ and $\mathrm{Cl}$ to $\mathrm{Au}_{16}$ cluster isomers are rather large, the distortions in their geometry due to tagging are

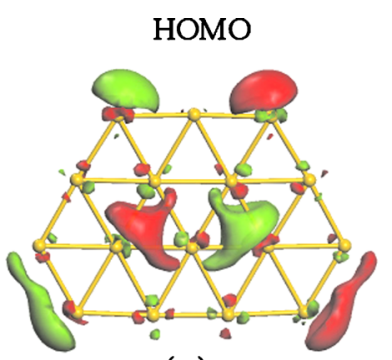

$\left(a_{1}\right)$

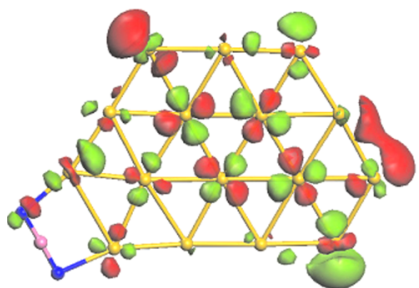

$\left(a_{2}\right)$

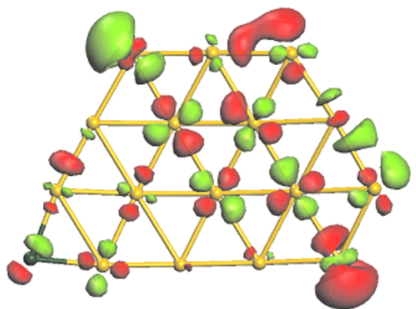

$\left(a_{3}\right)$

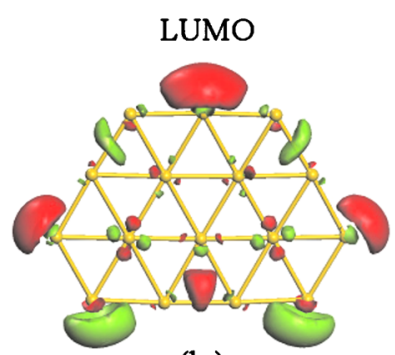

$\left(b_{1}\right)$

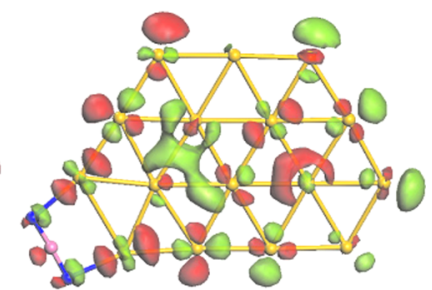

$\left(\mathrm{b}_{2}\right)$

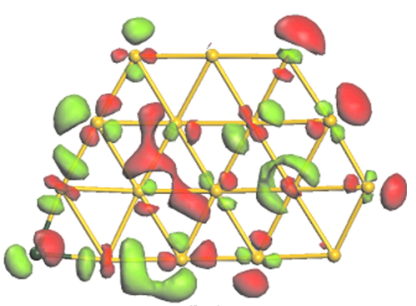

$\left(b_{3}\right)$
FIG. 3. (Color online) $\left(a_{1}\right),\left(a_{2}\right)$, and $\left(a_{3}\right)$ are the HOMO; $\left(b_{1}\right),\left(b_{2}\right)$, and $\left(b_{3}\right)$ are the LUMO for the planar configurations of the anionic clusters: $\mathrm{Au}_{16}{ }^{-}$, $\mathrm{Au}_{16} \mathrm{BO}_{2}^{-}$, and $\mathrm{Au}_{16} \mathrm{Cl}^{-}$, respectively.

rather minimal. We hope that this finding will stimulate future experimental studies.

This work was partially supported by grants from the U.S. Department of Energy and the National Natural Science Foundation of China (Grant No. NSFC-10874007).

${ }^{1}$ M. Ji, X. Gu, X. Li, X. G. Gong, J. Li, and L. S. Wang, Angew. Chem., Int. Ed. 44, 7119 (2005).

${ }^{2}$ J. Li, X. Li, H. J. Zhai, and L. S. Wang, Science 299, 864 (2003).

${ }^{3}$ S. Bulusu, X. Li, L. S. Wang, and X. C. Zeng, Proc. Natl. Acad. Sci. U.S.A. 103, 8326 (2006).

${ }^{4}$ X. Xing, B. Yoon, U. Landman, and J. H. Parks, Phys. Rev. B 74, 165423 (2006)

${ }^{5}$ F. Furche, R. Ahlrichs, P. Weis, C. Jacob, S. Gilb, T. Bierweiler, and M. M. Kappes, J. Chem. Phys. 117, 6982 (2002).

${ }^{6}$ S. Gilb, P. Weis, F. Furche, R. Ahlrichs, and M. M. Kappes, J. Chem. Phys. 116, 4094 (2002).

${ }^{7}$ M. P. Johansson, A. Lechtken, D. Schooss, M. M. Kappes, and F. Furche, Phys. Rev. A 77, 053202 (2008).

${ }^{8}$ G. Chen, Q. Wang, Q. Sun, and P. Jena, J. Chem. Phys. 132, 194306 (2010).

${ }^{9}$ J. Wang, G. Wang, and J. Zhao, Phys. Rev. B 66, 035418 (2002).

${ }^{10}$ S. Bulusu and X. C. Zeng, J. Chem. Phys. 125, 154303 (2006).

${ }^{11}$ W. Huang and L. S. Wang, Phys. Rev. Lett. 102, 153401 (2009).

${ }^{12}$ M. Götz, M. Willis, A. K. Kandalam, G. Ganteför, and P. Jena, ChemPhysChem 11, 853 (2010).

${ }^{13}$ H. J. Zhai, L. M. Wang, S. D. Li, and L. S. Wang, J. Phys. Chem. A 111, 1030 (2007).

${ }^{14}$ Y. Wang and J. P. Perdew, Phys. Rev. B 44, 13298 (1991).

${ }^{15}$ G. Kresse and J. Joubert, Phys. Rev. B 59, 1758 (1999).

${ }^{16}$ G. Kresse and J. Furthmüller, Phys. Rev. B 54, 11169 (1996).

${ }^{17}$ R. W. F. Bader, A Quantum Theory (Oxford University Press, Oxford, 1990).

${ }^{18}$ G. Henkelman, A. Arnaldsson, and H. Jónsson, Comput. Mater. Sci. 36, 354 (2006). 